\title{
54. Kongress der Deutschen Gesellschaft für Pneumologie und Beatmungsmedizin e. V.
}

\author{
Anlässlich des diesjährigen DGP-Kongresses, der vom \\ 20.-23. März 2013 in Hannover stattfand, geben die \\ 15 wissenschaftlichen Sektionen in dieser und der vorherigen \\ Ausgabe der „Pneumologie“ einen aktuellen Überblick über \\ zurückliegende Aktivitäten, personelle und inhaltliche \\ Neuerungen sowie einen Ausblick auf kommende Termine \\ und Schwerpunkte in den jeweiligen Fachbereichen.
}

Sektion 7
Klinische
Pneumologie

Die vorrangige Aufgabe der Sektion Klinische Pneumologie fokussierte auf die Auswahl und Zusammenstellung der zahlreichen klinischen Symposien, Postgraduiertenkurse und Frühseminare für unseren Jahreskongress. Daneben waren die eingereichten Poster zu bewerten und für Posterbegehungen und freie Vorträge zusammenzustellen.

Beim letztjährigen Kongress in Nürnberg konnten von der Sektion 7 insgesamt 12 Symposien platziert werden, davon 2 Veranstaltungen im Forum Fort- und Weiterbildung. Die Symposien waren alle sehr gut besucht mit z.T. über 200 Besuchern (Pulmonale Intoxikationen), ebenso die beiden Frühseminare und der Postgraduiertenkurs. Bei insgesamt 6 Veranstaltungen wurden Kooperationen mit anderen Sektionen hergestellt.

Für den diesjährigen DGP-Kongress konnten wieder 8 Symposien ausschließlich durch die Sektion 7 platziert werden, davon 3 Veranstaltungen im ILD-Track. Insgesamt 20 weitere Veranstaltungen wurden in Kooperation mit anderen Sektionen zusammengestellt. Eingebracht wurden des weiteren 2 Frühseminare (1-mal in Kooperation) und 9 Postgraduiertenseminare (7-mal in Kooperation). Für unsere Sektion wurden insgesamt 78 Poster ein- gereicht, mit denen 2 Sitzungen für freie Vorträge und insgesamt 5 Posterbegehungen organisiert werden konnten.

Mitglieder der Sektion Klinische Pneumologie sind wesentlich an der Durchführung und Auswertung der Daten des COSYCO-Netzes beteiligt, dem Kompetenznetz für obstruktive Atemwegserkrankungen, Asthma und COPD des BMBFs. Hier werden regelmäßig „Investigator Meetings" ausgerichtet.

Wie alljährlich wurde auch im vergangenen Jahr das Bad-Reichenhaller-Kolloquium in Kooperation mit der DGP von Mitgliedern der Sektion 7 und 12 mit dem Hauptthema Lunge-Umwelt-Arbeit veranstaltet. Knapp 600 Teilnehmer konnten registriert werden. Auch für das Jahr 2013 ist das Bad-Reichenhaller-Kolloquium bereits wieder vollständig organisiert mit einem Expertenforum zur Thematik COPD. Die besonders gute und intensive Zusammenarbeit mit der Deutschen Atemwegsliga ebenso wie mit der Sektion 12, Prävention und Rehabilitation, ist hier hervorzuheben.

In der Fachzeitschrift „Pneumologie“ wurden im Jahr 201216 Artikel von Mitgliedern der Sektion 7 oder unter deren Beteiligung publiziert - u. a auch die S1Leitline „Nosokomiale Pneumonie“.

Prof. Rainer W. Hauck, Bad Reichenhall und Prof. Jürgen Behr, München
Sektion 9

\section{Pädiatrische Pneumologie}

Passend zum Thema „Transition“ des DGP-Kongresses 2013 entstehen Leitlinien zur „Diagnose und Behandlung der Mukoviszidose", die wichtige Werkzeuge bei der integrativen Versorgung von Patienten mit Cystischer Fibrose aller Altersgruppen sein werden. Weiterhin wurde ein Referenzzentrum für Ataxia Teleangiectasia in Frankfurt gegründet und ein Forschungsprojekt Bronchiolitis obliterans initiiert, das mithilfe des Deutschen Lungenregisters Erkenntnisse über Häufigkeit, Ursache und Pathophysiologie der chronischen bronchialen Entzündung im Kindes- und Jugendalter gewinnen soll.

In Kooperation mit der Deutschen Gesellschaft für Pädiatrische Infektiologie (DGPI), Pädiatrische Pneumologie (GPP), Pädiatrische Kardiologie (DGPK), Neonatologie und pädiatrische Intensivmedizin (DNPI) wurde 2012 die Leitlinie zur Passiv-Immunprophylaxe von Erkrankungen durch das Respiratory Syncytial Virus, einer lebensbedrohlichen Atemwegsinfektion des frühen Kindesalters, aktualisiert. Hierdurch wird künftig die Berücksichtigung bislang unterversorgter Patientengruppen (z.B. neuromuskuläre Erkrankungen) verbessert, gleichzeitig aber auch der kostenbewusste Umgang mit dieser hochpreisigen Ressource optimiert. Die länderübergreifende (Deutschland, Österreich, Schweiz) Leitlinie „Management der ambulant erworbene Pneumonie bei Kindern und Jugendlichen" in Kooperation mit der Paul-Ehrlich-Gesellschaft, der DGPI und der GPP macht gute Fortschritte.

Forschungsglanzlichter in der pädiatrischen Pneumologie waren 2012 u.a. Arbeiten zur fetalen/frühkindlichen Programmierung des allergischen Asthmas (PD Dr. Bianca Schaub, München), Genom-gestützte Untersuchungen zur Immunregulation bei Mukoviszidose (Prof. Dominik Hartl, Tübingen, der mit der Cournand Lecture bei der ERS Tagung in Wien ausgezeichnet wurde), Studien zur T-Zell-Differenzierung und Regulation bei Asthma (Prof. Gesine Hansen, Hannover) und epigenetische Analysen (Prof. Michael Kabesch, Regensburg) zu Umweltbedingungen bei Asthma und Allergie. Prof. Eri- 
ka von Mutius, München, erhielt den Leibniz-Preis der Deutschen Forschungsgemeinschaft.

Die Bundesarbeitsgemeinschaft pädiatrischer Pneumologen (BAPP) hat ihre Aktivitäten weiter ausgebaut; finalisiert wurde u.a. ein Qualitätsmanagement-Handbuch für kinderpneumologische Standards, das auch für internistische Pneumologen eine Bereicherung darstellen wird.

Prof. Markus A. Rose, Frankfurt a.M.

\section{Sektion 10}

\section{Pathophysiologie und Aerosolmedizin}

Die Aktivitäten der Sektion bieten im Jahresverlauf mittlerweile 3 Schwerpunkte. Am 2. und 3. Oktober 2012 fand die Herbsttagung der Sektion in Verbindung mit der Deutschen Physiologischen Gesellschaft (Sektion Klinische Physiologie, Leitung Prof. Thorsten Schäfer, Bochum) statt und war mit 30 Teilnehmern gut besucht. Hauptthema war die Diskussion von Strategien, die neuen SpirometrieNormwerte einzuführen. Es war eine große Ehre, dass Prof. Philip H. Quanjer, Leiden, trotz angegriffenem Gesundheitszustands ein Übersichtsreferat zu den von ihm maßgeblich erarbeiteten Spirometrie-Normwerten der „Global lung function-Initiative“ hielt. Weitere Diskussionspunkte waren Gasauswaschverfahren in der Lungenfunktionsdiagnostik, Pers- pektiven der Exhalat-Diagnostik und pathophysiologische Veränderungen der Lunge, abgeleitet aus den Ergebnissen einer Weaning-Datenbank. Die Herbsttagung 2013 wird voraussichtlich Anfang Oktober in Kiel stattfinden.

Das 17. Deutsche Aerosol-Therapie-Seminar in Zusammenarbeit mit der „International Society for Aerosols in Medicine“ (ISAM) fand am 2. und 3. November in Marburg statt. Mit über 100 Teilnehmern war ein wichtiges Schwerpunktthema „Kleine Atemwege“. Pathologische Aspekte sowie die Möglichkeiten der lungenfunktionellen Diagnostik und der Bildgebung wurden ausgiebig diskutiert. Spannend war auch ein Referat zur vergleichenden Anatomie in der Tierlunge.

Am 1. Dezember 2012 wurde zum Thema Atemmuskeltraining das erste IMT-Symposium in Frankfurt veranstaltet. Bereits wenige Zeit nach Eröffnung der Anmeldung lagen weit mehr als 100 Anmeldungen für die vorgesehenen max. 100 Teilnahmeplätze vor, so dass die Kapazität schließlich auf 130 aufgestockt wurde. Leider konnten hiernach immer noch viele Interessenten aus begrenzten Kapazititätsgründen, insbesondere der Workshop-Anteile des Symposiums nicht teilnehmen. Aufgrund der außerordentlich hohen Nachfrage und des sehr positiven Feedbacks der Teilnehmer ist für den 16. November 2013 eine Neuauflage des IMTSymposiums in Frankfurt vorgesehen.

Die Arbeitsgruppe Sauerstofftherapie wurde aufgelöst und unter dem Dach der Sektion eingegliedert.

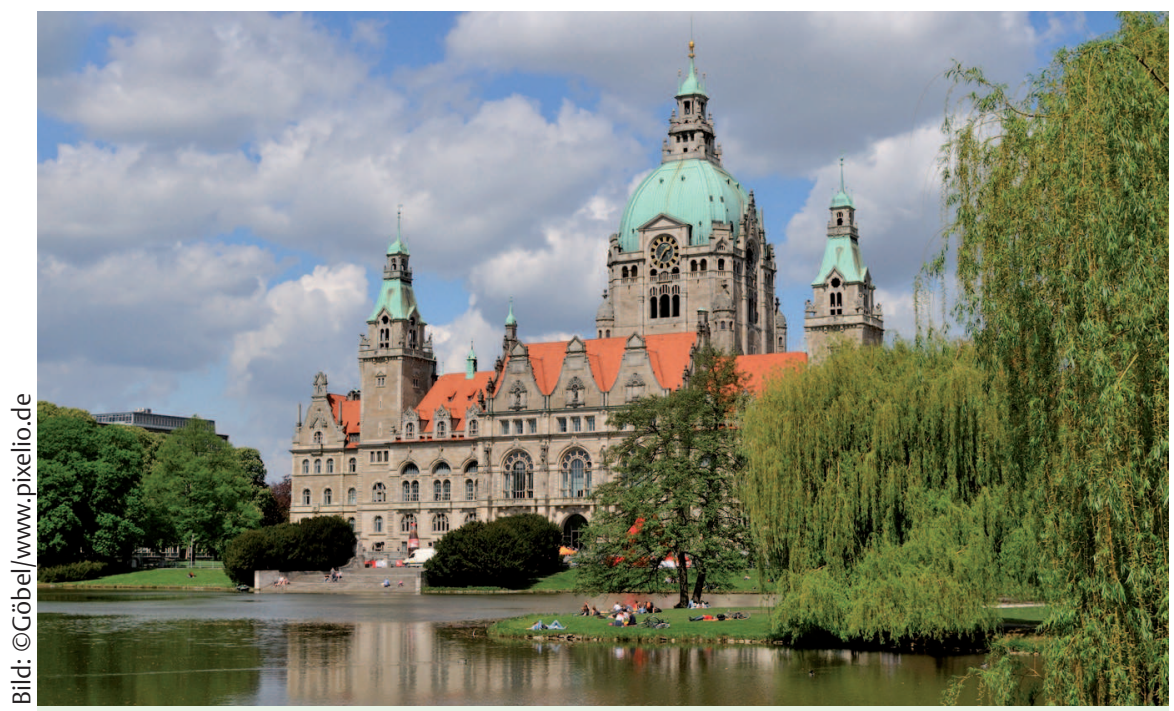

Rund 3300 Pneumologen trafen sich auf der 54. Jahrestagung der DGP im März 2013 in der niedersächsischen Landeshauptstadt Hannover.
Anlässlich der DGP-Tagung 2013 in Hannover wurden von der Sektion 4 Symposien, 2 Postgraduiertenkurse und der traditionelle Workshop des Arbeitskreises „Respiratorisches System“ der Deutschen Veterinärmedizinischen Gesellschaft ausgerichtet. Weiterhin ist die Sektion auch an zahlreichen anderen Symposien thematisch beteiligt.

Die Homepage der Sektion wurde überarbeitet und aktualisiert. Mitglieder der Sektion sind in die Überarbeitung der Leitlinie zum Asthma bronchiale sowie in die Neuerstellung der Leitlinie zur Spirometrie eingebunden.

Mitglieder der Sektion waren an Publikationen u.a. in der Zeitschrift „Pneumologie“ zum Thema Diagnostik und Therapie der Bronchiolitis sowie Diagnostik und Therapie der peripheren Atemwege beteiligt. Im Rahmen der durch die Deutsche Forschungsgemeinschaft (DFG) geförderten Projekte wurden auf dem Gebiet der Atemphysiologie neue Erkenntnisse erzielt und u.a. eine Originalarbeit zur Atemmuskelfunktion bei Lungenfibrose im „European Respiratory Journal“ publiziert. Im Herbst 2013 wird PD Dr. Hans-Joachim Kabitz, Freiburg, an einer groß angelegten schweizerisch-deutschen Forschungsexpedition im Himalaya teilnehmen und hier atemphysiologische Messungen bis auf Höhen von ca. 6900 m über NN durchführen.

Dr. Peter Haidl, Schmallenberg und PD Dr. Hans-Joachim Kabitz, Freiburg

\section{Pneumologische} Onkologie

Die Sektion 11 war 2012 intensiv an der wissenschaftlichen Gestaltung des Jahreskongresses der Gesellschaft mit der Durchführung von Symposien, Postgraduiertenkursen, Frühseminaren, Vortrags- und Postersitzungen alleine und in Kooperation mit anderen Sektionen beteiligt.

Während der jährlichen Sektionssitzung wurde zum einen das Programm für den nächsten Kongress 2013 in Hannover besprochen und beschlossen und zum anderen wichtige Prozesse wie die Entwicklung eines Ausbildungscurriculums für die 
pneumologische Onkologie, das den Kriterien der DGP und DKG entspricht und für den Zertifizierungsprozess zum Lungenkrebszentrum genutzt werden soll, diskutiert. Es ist geplant, dieses Curriculum in 3 Teilen durchzuführen unter Einbeziehung eines PG-Kurses beim DGP-Kongress und des jährlichen POA/DGP-Symposiums. Hier wird eine Abstimmung mit Dr. Orthrud Karg erfolgen, die neu benannte Leiterin der Fortbildungsakademie der DGP.

Am 11. Mai 2012 und am 12. Oktober 2012 fanden das 4. bzw. 5. INAT-Treffen (INAT: Interdisziplinären Austausch in der Thoraxonkologie) in Heidelberg statt. Im großen Rahmen wurden ambulante Versorgungsstrukturen, Qualifizierung durch Weiterbildung im Kontext von Lungenkrebszentren sowie Versorgungs- und Entscheidungslinien in palliativer Behandlungssituation diskutiert.

Darüber hinaus fand unter maßgeblicher Beteiligung der Sektion 11 in Zusammenarbeit mit der Pneumologisch-Onkologischen Arbeitsgemeinschaft (POA), der Arbeitsgemeinschaft internistische Onkologie (AIO) und der Arbeitsgruppe onkologische Thoraxchirurgie (AOT) und der Arbeitsgemeinschaft radiologische Onkologie (ARO) ein interdisziplinäres Symposium zur Thoraxonkologie am 8. und 9. Februar 2013 in der Thoraxklinik Heidelberg statt. Kernthemen auf diesem Symposium waren die Radioonkologie, supportive Maßnahmen und ein Update zu aktuellen Therapieverfahren und Therapieergebnissen in der Thoraxonkologie.

Die Präsenz der pneumologischen Onkologie wurde durch Mitarbeit von etlichen Sektionsmitgliedern in nationalen und internationalen Arbeitsgruppen wahrgenommen u.a. in der ERS, IASLC, ESMO und ASCO. Prof. Rudolf M. Huber, München, nimmt das Amt des Vertreters der DGP in der AIO wahr. Prof. Michael Thomas, Heidelberg, und Dr. Karl-Matthias Deppermann, Erfurt, sind Vorsitzender und stellvertretender Vorsitzender der POA. PD Dr. Martin Reck, Großhansdorf, ist Mitglied des „Chest Committees" in der ESMO und Chair des „Membership Committees“ der IASLC. Neben der wissenschaftlichen Vorbereitung von internationalen Kongressen war die Sektion 11 ebenfalls an der Erstellung von internationalen Empfehlungen wie den „ESMO Recommendations und Consensus Statements“ in 2012 beteiligt.

Dr. Andreas Gröschel, Aachen

\section{Sektion 12}

\section{Prävention und Rehabilitation}

Auch im Jahr 2012 wurden die traditionellen Fortbildungsveranstaltungen der Sektion durchgeführt. Im Rahmen des 44. Bad Reichenhaller Kolloquiums vom 15.17. Juni hat die Sektion erfolgreich den 12. Bad Reichenhaller Workshop mit dem Thema „Rehabilitation bei COPD - die Komorbidität im Focus“ abgehalten. Im Oktober 2012 fand erneut eine internationale Fortbildung im Rahmen einer D.A.CHTagung mit relevanter Beteiligung Deutscher Redner in Salzburg statt. Dies stellt inzwischen eine an Kontinuität gewinnende, grenzüberschreitende Veranstaltung dar, die von Vertretern der Rehabilitation aus Österreich, der Schweiz und aus Deutschland initiiert wurde.

Während des 53. Kongresses der Deutschen Gesellschaft für Pneumologie und Beatmungsmedizin 2012 in Nürnberg wurden in Zusammenarbeit mit anderen Sektionen und Arbeitsgemeinschaften 2 Postgraduiertenkurse, 6 Symposien und 2 Posterbegehungen durchgeführt. Die Zusammenarbeit mit anderen Sektionen (wie z.B. Klinische Pneumologie, Pneumologische Onkologie), Arbeitsgemeinschaften (Palliativmedizin) und dem Bundesverband der Pneumologen funktioniert mit Blick auf die Einbindung von Rednern aus dem Bereich Rehabilitation auch sehr gut. Gleiches gilt für die Kooperation mit der AG Atemtherapie im Zentralverband der Krankengymnasten. Die Sektion 12 hatte erneut die Möglichkeit, das Physiotherapieseminar mitzugestalten. Somit konnte sich die Sektion Rehabilitation erneut als aktive Gruppe präsentieren. Erfreulicherweise werden die nicht medikamentösen Therapiemaßnahmen, also das zentrale Thema der Sektion, mit zunehmender Bedeutung wahrgenommen.

\section{Publikationen \\ $\nabla$}

Als sehr bemerkenswert ist die Zunahme an Publikationen von bzw. unter Mitarbeit von Mitgliedern der Sektion 12 zu bezeichnen. So wurden in internationalen Journals insgesamt 8 Arbeiten publiziert, was es in diesem Ausmaß zuvor noch nie gegeben hatte. Für deutschsprachige Zeitschriften mit Review-Verfahren sind 7
Publikationen zu nennen. Zudem sind 5 Berichte zu verschiedenen Themen in einem DRV-Schriftband zu erwähnen.

In der Rubrik „The Best Posters“ beim Meeting der „European Respiratory Society" in Wien 2012 waren immerhin 5 eingereichte Beiträge von Mitgliedern der Sektion 12 bzw. zu Themen der Pneumologischen Rehabilitation berücksichtigt worden.

Im Jahr 2012 wurde erneut ein Preis verliehen. So ging der Wissenschaftspreis (1. Preis; bundesweite Ausschreibung für Bachelor- und Master-Abschlussarbeiten) des IFK (Bundesverband selbstständiger Physiotherapeuten) an Stella Seeberg von der Schön Klinik Berchdesgardener Land für ihre Arbeit zum Thema „Der Effekt von reflektorischer Atemtherapie im Vergleich zu standard-atemphysiotherapeutischen Maßnahmen auf Verringerung der Dyspnoe und Steigerung der Aktivität bei COPD-Patienten“.

\section{Gesundheitspolitische Aktivitäten \\ $\nabla$}

Unter Mitwirkung des DGP-Präsidenten, Prof. Heinrich Worth, sowie des Sektionssprechers, Dr. Klaus Kenn, fand am 8. Juni 2012 ein Treffen mit den zuständigen Leiterinnen für die Bereiche Rehabilitation

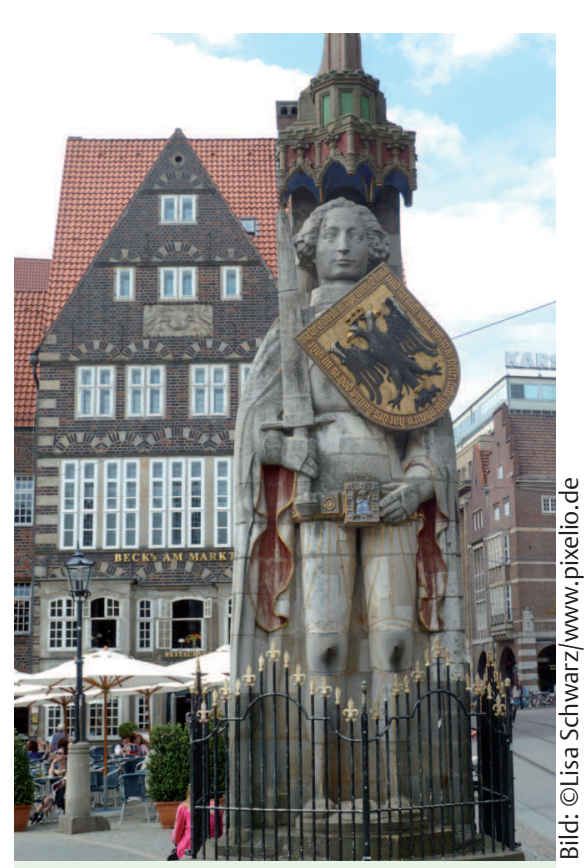

Der Bremer Roland, eine 1404 errichtete Statue auf dem Marktplatz vor dem Rathaus, ist ein Wahrzeichen der Hansestadt Bremen, die im kommenden Jahr Veranstaltungsort des 55. DGP-Kongresses sein wird. 
der Bundes-AOK sowie des Bundesverbandes der Ersatzkassen in Berlin statt.

Die Vorstellung der Datenlage zur Pneumologischen Rehabilitation, insbesondere die aktuelle Cochrane-Analyse und der daraus abgeleitete positive Kommentar des IQWiG wurde mit großem Interesse aufgenommen, wenngleich rasch klar wurde, dass ein einzelnes Gespräch zu keinerlei Strukturänderungen führen wird. Einzig für den Bereich Lungensport konnte rasch dahingehend Übereinstimmung erzielt werden, dass hier Verbesserungen einer pneumologisch-spezifischen Ausbildung sinnvoll sind und von den Kassen unterstützt werden sollen. Prof. Heinrich Worth, Fürth, kann ggf. über weitere Verhandlungen in den zuständigen Gremien berichten.

Die Notwendigkeit zum Auf- und Ausbau ambulanter Reha-Strukturen konnte zwar dargestellt werden, konkrete Ansätze zur Realisierung ließen sich aber nicht erarbeiten. Es stellt sich prinzipiell die Frage, ob zum Thema COPD nicht seitens der DGP ein Vorstoß in Richtung Kostenträger erfolgen sollte. Dies hätte zum Ziel, proaktiv eine kompetente, möglichst facharztgesteuerte COPD-Versorgungsstruktur vorzuschlagen, die zulässt, dass sich der Patient je nach Krankheitsausprägung und aktueller Problemkonstellation an der richtigen Stelle in der definierten Versorgungskette befindet. Die Kassen haben offenbar die Tragweite der Diagnose „COPD“ realisiert und sind ggf. für solche Initiativen offen.

Dr. Klaus Kenn, Schönau, und Dr. Karsten Siemon, Schmallenberg

\section{Sektion 13}

\section{Thoraxchirurgie}

Die Sektion hat dieses Jahr im Rahmen des 54. Kongresses der DGP 19 Sitzungen aktiv mitgestaltet, darunter 1 Frühseminar, 13 Symposien, 1 Seminar und 1 Postgraduiertenkurs. Mit dieser konstant hohen Beteiligung der Sektion wird die enge klinische und wissenschaftliche Zusammenarbeit von Pneumologie und Thoraxchirurgie deutlich.

Die Sektion Thoraxchirurgie führt kein wissenschaftliches Eigenleben, da die Mitglieder der Sektion in der Regel gleichzeitig Mitglieder der Deutschen Gesellschaft für Thoraxchirurgie sind und sich dort wissenschaftlich engagieren. Die Sektion versteht sich als interdisziplinärer Partner für die Pneumologie. Schwerpunkt unserer Arbeit ist die Gestaltung interdisziplinärer Veranstaltungen für die Fort- und Weiterbildung.

Die Sektion Thoraxchirurgie zählt aktuell 103 Mitglieder mit aktivem und passivem Stimmrecht - ein deutlicher Zuwachs im Vergleich zum Jahr 2011 mit 84 Mitgliedern.

Beim 54. DGP-Kongress in Hannover wurden turnusgemäß Sprecher und stellvertretender Sprecher der Sektion neu gewählt. Zum Sprecher wurde der bisher stellvertretende Sprecher Dr. Stephan Eggeling, Berlin, und zum neuen stellvertretenden Sprecher Dr. Erich Hecker, Herne, gewählt.

\section{Sektion 14}

\section{Zellbiologie}

Die Sektion Zellbiologie hat zum Ziel, die Grundlagenforschung innerhalb der DGP zu fördern und für die klinische Anwendung nutzbar zu machen. Das Ziel ist insbesondere, junge Forscher für die Pneumologie zu gewinnen und junge Ärzte für die Forschung zu begeistern. Angesichts der Veränderungen in der klinischen Pneumologie (Arbeitsverdichtung, Spezialisierung, Generation Y) sind dies Ziele höchster Priorität, die gleichzeitig mehr Engagement erfordern, um erreicht zu werden. Im vergangenen Jahr lag die Arbeit der Sektion auf folgenden Schwerpunkten:

- Hauptkongress: Reine Grundlagensymposien machen wenig Sinn, da sich das Auditorium des Kongresses eher für die klinische Aus- und Weiterbildung interessiert. Hier war das Ziel, Inhalte der Grundlagenforschung in die Schwerpunktthemen der Kongresse 2012 und 2013 zu integrieren. Die Sektion vergab Reisestipendien zum Hauptkongress für qualifizierte Grundlagenforscher.

- Die Weiterentwicklung des Herbsttreffens, das nun seit 2012 zusammen mit der Sektion Infektiologie und Tuberkulose durchgeführt wird. Diese Treffen sind ein großer Erfolg und stellen insbesondere für junge Forscher ein Forum dar. Das Ziel ist es daher, die „Herbsttagung“ weiter als grundlagenwissenschaftliches Forum in der DGP auszubauen. Neben den beiden genannten Sektionen soll diese auch allen anderen Sektionen offenstehen.

Prof. Robert Bals, Homburg/Saar

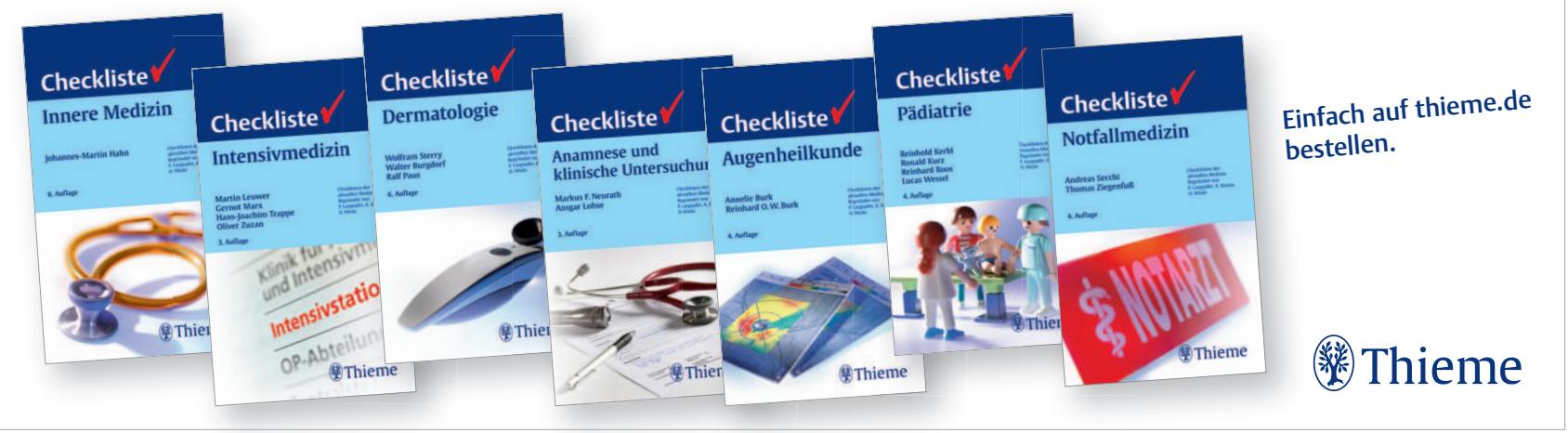


Sektion 15

\section{Medizinische Assistenzberufe}

Erfreulicherweise haben auch weiterhin immer mehr in der Pneumologie und Beatmungsmedizin tätige Angehörige der medizinischen Assistenzberufe Interesse, sich in einer Fachgesellschaft zu organisieren, was durch das Wachstum der Sektion 15 auf eine Mitgliederzahl von 159 zu belegen ist. Die hier vertretenen Berufsgruppen sind hauptsächlich Atmungstherapeuten, Physiotherapeuten und Pflegekräfte. Der Beitritt auch anderer, maßgeblich an der therapeutischen Versorgung pneumologisch erkrankter Patienten beteiligten Berufsgruppen, wie z. B. Logopäden, wird sehr begrüßt.

Im Jahr 2012 war die Sektion Medizinische Assistenzberufe wie bereits in den Vorjahren am 7. Beatmungssymposium unter Schirmherrschaft der DGP im Rahmen der 20. Jahrestagung der Deutschen Interdisziplinären Gesellschaft für Außerklinische Beatmung e.V. (DIGAB) vom 26.-28. April in Kassel beteiligt. Mitgestaltet wurden u.a. Workshops, Diskussionsforen und Symposien.

In Göttingen jährte sich zum 5. Mal die inzwischen zu einer festen Tradition gewordene gemeinsame Herbsttagung der Sektionen 5 Intensiv- und Beatmungsmedizin und 15 Medizinische Assistenzberufe vom 2.-3. November. Auf dieser Veranstaltung gab es erstmals 2 gemeinsame Symposien mit Referenten aus beiden Sektionen zum Thema „Atemmuskulatur und Beatmung“ und „Management des beatmeten Patienten“. Am Nachmittag fand als Parallelveranstaltung für die Sektion 15 ein Seminar über „Wissenschaftliches Arbeiten für Einsteiger“ statt.

Die Bedeutung der multiprofessionellen Zusammenarbeit in der Patientenversorgung zeigt sich auch durch die stetig wachsende aktive Beteiligung der medizinischen Assistenzberufe am Programm des alljährlichen Jahreskongresses der DGP. So war die Sektion 15 in diesem Jahr federführend bei 2 Postgraduiertenkursen und Frühseminaren, 3 Symposien sowie bei 11 weiteren Veranstaltungen in Kooperation mit anderen Sektionen stark vertreten. Die Inhalte umfassten das ganze Spektrum der Pneumologie bis zu berufspolitisch bedeutsamen Themen.
Auf dem Sektionstreffen im Rahmen des diesjährigen DGP-Kongresses kam es vorzeitig zur Wahl des stellvertretenden Sektionssprechers, nachdem Kathrin Süß aus Gauting leider aus nachvollziehbaren Gründen von ihrem Amt zurücktreten musste. Die Sektion Medizinische Assistenzberufe freut sich sehr, Petra Will aus Münnerstadt (Atmungstherapeutin mit Kernkompetenz Physiotherapie) als neue stellvertretende Sprecherin begrüßen zu dürfen.

Hervorzuheben ist ebenfalls die fortbestehend große aktive Beteiligung an weiteren Kongressen und Tagungen diverser Fachgesellschaften und -verbände wie z.B. dem Kongress der Deutschen interdisziplinären Vereinigung für Intensiv- und Notfallmedizin (DIVI) sowie die Mitgestaltung von zahlreichen Fort- und Weiterbildungsveranstaltungen wie z.B. der Weiterbildung zum Atmungstherapeuten (DGP) oder der Fortbildungsreihe Atemphysiotherapie.

Darüber hinaus arbeitete die Sektion 15 an der Aktualisierung der Leitlinie zur Diagnostik und Therapie von Patienten mit Asthma und der Aktualisierung der nationalen Versorgungsleitlinie COPD mit und veröffentlichte Artikel in vielfältigen Fachzeitschriften wie z.B. dem Sonderheft der DIVI zum Thema Beatmung oder der Sonderausgabe der Zeitschrift „Atemwegs- und Lungenkrankheiten“ erschienen zum DGP-Kongress letzten Jahres.

In der im November 2010 gegründeten AG Atmungstherapeuten gab es im Rahmen des diesjährigen DGP-Kongresses turnusmäßige Neuwahlen von AG-Sprecher sowie Stellvertreter. Als Sprecherin wurde mit großer Freude erneut Dorit Schimandl aus Bad Berka bestätigt sowie als ihr neuer Stellvertreter Sören Tiedemann aus Hamburg, den die AG und die Sektion Medizinische Assistenzberufe herzlich im Amt begrüßt.

Die AG Atmungstherapeuten veranstaltete in Hannover das abschließende 3. Modul des ersten AT-Refresherkurses, welcher auch in den kommenden Jahren weitergeführt werden soll, um den Atmungstherapeuten ein festes Forum für Fortund Weiterbildung zu bieten.

Der bis dato sogenannte Atmungstherapeuten-Tag, ein jährliches Treffen der Atmungstherapeuten veranstaltet von der AG Atmungstherapeuten in Kooperation mit der Sektion 15, wurde in diesem Jahr umbenannt in die Jahrestagung der Atmungstherapeuten (DGP) und findet am 16. November 2013 in Münnerstadt statt.

Im letzten Jahr kamen mit Essen und Heidelberg 2 weitere Ausbildungsstätten für die Weiterbildung zum Atmungstherapeuten zu den bereits bestehenden 6 Zentren hinzu; mit Berlin folgt in diesem Jahr ein zusätzliches. Das Interesse und der Bedarf an der Weiterbildung zum Atmungstherapeuten nimmt weiter $\mathrm{zu}$, die Zahl der ausgebildeten Atmungstherapeuten in Deutschland beläuft sich aktuell auf sagenhafte 266 .

\section{Janine Wagner, Berlin}

AG Nachwuchsförderung

\section{Forum für junge Pneumologen}

Die AG Nachwuchsförderung hat wie es der Name schon sagt zum Ziel, junge Pneumologen zu fördern, indem aktuelle Problemfelder aufgezeigt und Lösungen diskutiert werden. Ein weiteres wichtiges Ziel ist es, Studierende und junge Ärzte für die Pneumologie zu begeistern. Angesichts der Veränderungen in der klinischen Pneumologie (Arbeitsverdichtung, Spezialisierung, Generation Y) sind dies Ziele höchster Priorität, die gleichzeitig immer mehr Engagement erfordern, um erreicht zu werden.

Im vergangenen Jahr hatte die Arbeit der AG folgende Schwerpunkte:

- Auf den Kongressen 2012 und 2013 wurden Symposien zu den Themen Nachwuchs und Generation Y veranstaltet, die gut besucht waren und zu intensiven Diskussion inspiriert haben.

- Die Internetseite www.ich-werdepneumologe.de wurde entwickelt und ist nun online. Dieses Portal wird kontinuierlich weiter entwickelt.

- Nachwuchsförderung ist kommunikativ und interdisziplinär, es wurde ein intensiver Diskurs mit anderen Arbeitsgemeinschaften, Sektionen und Organisationen (AG Aus-/Weiterbildung, AG Pneumologinnen, BDP u.a.) vorangetrieben.

Prof. Robert Bals, Homburg/Saar 